\title{
Successful introduction of robotic-assisted percutaneous coronary intervention system into Japanese clinical practice: a first-year survey at single center
}

\author{
Kotaro Kagiyama ${ }^{1} \cdot$ Yoshiaki Mitsutake $^{1} \cdot$ Takafumi Ueno $^{1,2} \cdot$ Shinji Sakai $^{3} \cdot$ Takuya Nakamura $^{4} \cdot$ Kazunori Yamaji $^{1}$. \\ Takashi Ishimatsu ${ }^{1} \cdot$ Masahiro Sasaki $^{1} \cdot$ Hidetoshi Chibana $^{1} \cdot$ Naoki Itaya $^{1} \cdot$ Ken-ichiro Sasaki $^{1} \cdot$ Yoshihiro Fukumoto $^{1}$
}

Received: 13 October 2020 / Accepted: 8 January 2021 / Published online: 27 January 2021

(c) Springer Japan KK, part of Springer Nature 2021

\begin{abstract}
In Japan, a robotic-assisted PCI (R-PCI) system, the CorPath GRX System (Corindus Inc.), has been approved for clinical use in 2018, which is the first introduction of R-PCI into Japan. In this study, the clinical performance of the R-PCI system in the initial year at Kurume University Hospital was evaluated comparing with conventional manual PCI (M-PCI). A total of 30 R-PCI and 77 M-PCI procedures performed between April 2019 and March 2020, were retrospectively included. The primary outcome was the rate of clinical success defined as $<30 \%$ residual stenosis without in-hospital major adverse cardiovascular events (MACE). The secondary outcomes were fluoroscopy time, dose area product (DAP), amount of radiation exposure to operators and assistants, procedural time, and contrast volume. Propensity-matching technique was used to match each R-PCI lesion to the nearest M-PCI lesion without replacement. After propensity score matching, $30 \mathrm{R}-\mathrm{PCI}$ procedures in 28 patients and $37 \mathrm{M}$-PCI procedures in 35 patients were analyzed. Clinical success rate with R-PCI was favorable and comparable to M-PCI (93.3 vs. 94.6\%, $p=0.97$ ), without any in-hospital MACE. The operator radiation exposure was significantly lower in R-PCI ( 0 vs. $24.5 \mu \mathrm{SV}, p<0.0001$ ). Radiation exposure to the patients was tended to be reduced by R-PCI (DAP: 77.6 vs. $\left.100.2 \mathrm{Gycm}^{2}, p=0.07\right)$. There were no statistically significant differences in radiation exposure to the assistant, fluoroscopy time, procedural time and contrast volume between the two groups (radiation exposure to the assistant: 10.5 vs. $10.0 \mu \mathrm{SV}$, $p=0.64$, fluoroscopy time: 27.5 vs. $30.1 \mathrm{~min}, p=0.55$, procedural time: 72.4 vs. $61.6 \mathrm{~min}, p=0.23$, and contrast volume: 93.2 vs. $102.0 \mathrm{ml}, p=0.36$ ). R-PCI in selected patients demonstrated favorable clinical outcomes with dramatical reduction of radiation exposure to operators.
\end{abstract}

Keywords Radiation exposure $\cdot$ Percutaneous coronary intervention · Robotic-assisted procedures

$\begin{array}{ll}\text { Abbreviations } \\ \text { CTO } & \text { Chronic total occlusion } \\ \text { DAP } & \text { Dose area product } \\ \text { ISR } & \text { In-stent restenosis } \\ \text { LAD } & \text { Left anterior descending artery }\end{array}$

Yoshiaki Mitsutake

mitsutake_yoshiaki@kurume-u.ac.jp

1 Division of Cardiovascular Medicine, Kurume University School of Medicine, 67 Asahi-machi, Kurume 830-0011, Japan

2 Fukuoka Memorial Hospital, Fukuoka, Japan

3 Center of Diagnostic Imaging, Kurume University Hospital, Kurume, Japan

4 Center of Clinical Engineering, Kurume University Hospital, Kurume, Japan
LCX Left circumflex artery

LMT Left main trunk

MACE Major adverse cardiac events

MI Myocardial infarction

M-PCI Manual percutaneous coronary intervention

PCI Percutaneous coronary intervention

QCA Quantitative coronary angiography

RCA Right coronary artery

R-PCI Robotic-assisted percutaneous coronary intervention

TVR Target vessel revascularization 


\section{Introduction}

Although several innovative devices related to percutaneous coronary intervention (PCI) have been developed, the fundamental operating style has not changed for long time. In conventional PCI, operators manipulate the devices under fluoroscopic guidance standing at the patients' tableside, while wearing heavy lead aprons and other radiation protection devices. These unfriendly environment to the operators is well known to be associated with operators' complications such as cancer, cataracts, orthopedic issues, and other medical problems [1-3].

A remote-controlled robotic-assisted system for PCI, the CorPath System (Corindus Inc., Waltham, MA, the United States) has been developed to address such limitations. Robotic-assisted PCI (R-PCI) enables operators manipulate PCI devices remotely sitting down at a radiation-shielded cockpit. In addition, R-PCI provides precise lesion measurement and improved device positioning, which may lead to reduce radiation exposure to patients as well as radiation exposure to operators.

In 2018, the second-generation R-PCI system, the CorPath GRX system (Corindus Inc.) has been approved for clinical use in Japan, which is the first introduction of R-PCI into Japan. We reported our first case series in Japan [4]. In this study, we evaluated the clinical performance of the R-PCI system in the initial year after introduction to our hospital, comparing with manual PCI (M-PCI) in the same time frame.

\section{Materials and methods}

This was a retrospective, single-center, non-randomized study. Between April 2019 and March 2020, a total of 30 robotic-assisted PCI (R-PCI) procedures in 28 patients were performed by 3 trained operators at Kurume University Hospital (operator A: 21 procedures, operator B: 7 procedures and operator $\mathrm{C}: 2$ procedures). The followings were the exclusion criteria to R-PCI in our center; patients with acute myocardial infarction (MI), chronic total occlusion (CTO) lesions, lesions expected to be required use of atherectomy device, and cardiogenic shock required mechanical support devises such as intra-aortic balloon pumping, extra-corporeal membrane oxygenation or temporary ventricular support pump catheter.

All patients were provided written informed consent prior to each PCI procedure. This study was approved by the Institutional Review Board of Kurume University (certification number: 20073) and followed the Declaration of Helsinki and the ethical standards of the responsible committee on human experimentation. Because this was a retrospective study, written informed consent form each enrolled patient was waived, instead of that, we provided an opportunity to opt out.

\section{Study outcomes and definitions}

The primary outcome was clinical success rate. Clinical success was defined as less than $30 \%$ residual stenosis determined by a quantitative coronary angiography (QCA), without major adverse cardiovascular events (MACE) either within $72 \mathrm{~h}$ of the procedure or before hospital discharge, whichever occurred first. MACE was defined as a composite of cardiac death, MI and clinically driven target vessel revascularization by percutaneous or surgical methods. MI was defined as (1) elevated creatine kinase myocardial band isoenzyme $(\mathrm{CK}-\mathrm{MB})>10$ times the upper limit of normal (ULN) or cardiac troponin (cTn) values $>70$ times ULN, or (2) CK-MB $>5$ times ULN or cTn values $>35$ times ULN with development of new pathological $\mathrm{Q}$ waves in two contiguous leads or left bundle branch block [5]. The secondary outcomes were procedural time, contrast volume, fluoroscopy time, dose area product (DAP), and amount of radiation exposure for main operator and assistant. Procedural time was defined as time during the engagement of guiding catheter into the coronary artery and the removal of the guiding catheter. To measure radiation exposure, the operator and the assistant wore an electronic pocket dosimeter (MYDOSE mini TM, ALOKA CO., LTD., Tokyo) on their left-side neck. Robotic technical success defined as clinical success and the completion of the PCI procedure entirely robotically or with partial manual assistance were also assessed. Manual assistance was defined as temporary disengagement of the robotic drive to use bedside manipulation of either the guide catheter, guidewire, or delivery system, with ultimate completion of the procedure using the re-engaged robotic drive. Manual conversion was defined as the disengagement of the robotic drive to use bedside manipulation of either the guide catheter, guidewire, or delivery system, which was required until the end of the procedure. Since intravascular imaging modality such as intravascular ultrasound or optical coherence tomography is incompatible with the current CorPath system, imaging devices need to be manipulated manually. In this study, the use of intravascular imaging modality is defined as "planned manual assistance" and is excluded from manual assistance.

\section{Robotic-assisted PCI system}

The details of the CorPath GRX system were described elsewhere [6-8]. Briefly, the CorPath GRX system consists of an interventional cockpit and a bedside unit (Fig. 1). The interventional cockpit is a radiation-shielded mobile workstation 

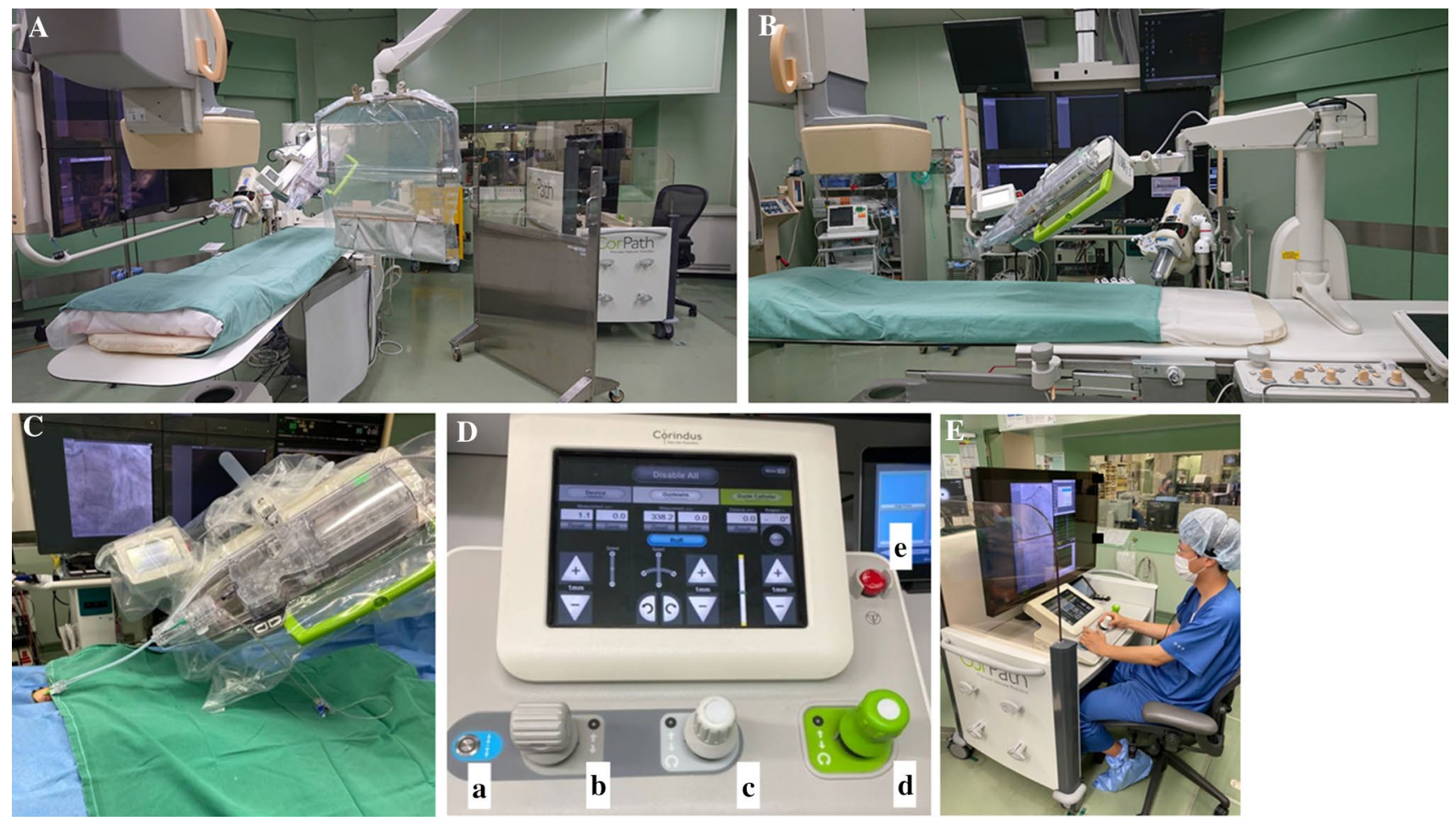

Fig. 1 The CorPath GRX system. a and b Overview of the CorPath GRX system in the catheterization laboratory. c A single-use cassette connected with a vascular access sheath. d Control console. a Turbo button. b Balloon/stent catheter joystick. c Guidewire joystick.

that contains a console with joysticks and touchscreen controls to control movement of the balloon/stent delivery system, guidewire, or guiding catheter. The bedside unit consists of a single-use cassette, articulating arm, and robotic drive. During the PCI procedure, the single-use cassette is mounted on a robotic drive and loaded with interventional equipment, which translates commands from the cockpit to independently manipulate each device. The system allows the operator to remotely control the movement of PCI devices by increments as small as $1 \mathrm{~mm}$ proximally or distally. In addition, the system allows the operator to measure lesion lengths during guidewire or balloon catheter movement. The system meets all commercial 0.014-inch guidewires and rapid-exchange balloon/stent catheters, and standard coronary guiding catheters of various sizes. Gaining a vascular access, advancement, and engagement of the guiding catheter were performed manually. The fluoroscopy is controlled by the seated operator, and contrast injection and exchange of devices in the cassette are performed by the tableside assistant.

\section{Statistical analysis}

Continuous variables are presented as the means with standard deviations or the median and interquartile range, d Guide catheter joystick. e Emergency stop button. e An operator remotely controls the movement of PCI devices, sitting down at a radiation-shielded cockpit

according to their normal or not normal distribution. Differences in continuous parameters were evaluated using an unpaired $t$-test or Mann-Whitney $U$ test. Categorical variables are presented as frequency counts and intergroup comparisons were made using Fisher's exact test or Chisquare test. To match lesion background between R-PCI and M-PCI, propensity score-matched analysis was performed. Binary logistic regression was performed to calculate the propensity score. Target vessel, the American College of Cardiology/American Heart Association (ACC/AHA) lesion classification, the presence of in-stent restenosis (ISR), small vessel lesion (less than $2.5 \mathrm{~mm}$ ), moderate to severe calcified lesion, tortuosity (more than moderate), diffuse lesion (over $20 \mathrm{~mm}$ ), and bifurcation lesion were included in the binary logistic regression to estimate the probability. A nearestneighbor greedy caliper match technique using caliper size one-quarter of the SD (caliper $=0.05$ ) was used to match each R-PCI patient to the nearest M-PCI patient without replacement. In the propensity score-matched cohort, comparisons were performed using unpaired $t$-test for numeric variables and Fisher's exact test or Chi-square test for categorical variables. All statistical analyses were performed using SPSS Statistics software (version 23.0, SPSS Inc., Chicago, IL, the United States). A $p$ value $<0.05$ was considered statistically significant. 


\section{Results}

Between April 2019 and March 2020, there were 217 PCI procedures at Kurume University Hospital. Of these, a total of 30 R-PCI procedures were performed (13.8\%). From 187 M-PCI, we excluded 80 emergent PCI procedures and 30 procedures because of CTO-PCI or use of atherectomy devices. Finally, we included 30 R-PCI procedures in 28 patients with 48 lesions and $77 \mathrm{M}$-PCI procedures in 73 patients with 108 lesions in this analysis (Fig. 2).

Table 1 demonstrates patients' background. There were no significant differences in clinical background between the R-PCI and the M-PCI. Regarding lesion characteristics, except the prevalence of bifurcation lesion, there were no significant differences in target vessel and prevalence of featured lesion such as calcified lesion, small vessel disease, diffuse lesion between the two groups (bifurcation lesion; R-PCI: $20.5 \%$ vs. M-PCI: $43.1 \%, p=0.047)$. In the both groups, more than $70 \%$ of lesions were treated with stent deployment and intracoronary imaging device was used in over $98 \%$ of lesions (Table 2).

Table 3 shows the study outcomes. Clinical success rate was $93.3 \%$ in the R-PCI and $92.2 \%$ in the M-PCI, respectively $(p=0.97)$. In the R-PCI, 2 procedures $(6.7 \%)$ required partial manual assistance due to difficulty of guidewire crossing $(n=1)$ and stent delivery $(n=1)$, and 3 procedures $(10.0 \%)$ were converted to manual procedure due to difficulty of guidewire crossing $(n=2)$ and balloon catheter delivery $(n=1)$. Two of the three R-PCI procedures required manual conversion resulted in failure even
Table 1 Comparison of patient characteristics between the R-PCI and the M-PCI

\begin{tabular}{llll}
\hline & R-PCI $(n=28)$ & M-PCI $(n=73)$ & $p$ value \\
\hline Age, years & $70.9 \pm 9.9$ & $73.0 \pm 11.3$ & 0.39 \\
Male sex & $75.0 \%(21)$ & $60.3 \%(44)$ & 0.25 \\
Prior PCI & $57.1 \%(16)$ & $43.8 \%(32)$ & 0.25 \\
Prior MI & $28.6 \%(8)$ & $16.4 \%(12)$ & 0.28 \\
Prior CABG & $10.7 \%(3)$ & $9.6 \%(7)$ & 1.0 \\
Heart failure & $35.7 \%(10)$ & $27.4 \%(20)$ & 0.56 \\
Diabetes mellitus & $60.7 \%(17)$ & $53.4 \%(39)$ & 0.66 \\
Hyperlipidemia & $67.9 \%(19)$ & $76.7 \%(56)$ & 0.51 \\
Hypertension & $75.0 \%(21)$ & $86.3 \%(63)$ & 0.29 \\
Prior stroke & $14.3 \%(4)$ & $15.1 \%(11)$ & 1.0 \\
PAD & $10.7 \%(3)$ & $13.7 \%(10)$ & 0.95 \\
Current smoker & $53.6 \%(15)$ & $42.5 \%(31)$ & 0.44 \\
CKD & $28.6 \%(8)$ & $35.6 \%(26)$ & 0.66 \\
Hemodialysis & $14.3 \%(4)$ & $8.2 \%(6)$ & 0.59 \\
\hline
\end{tabular}

All values are $\%(N)$ or mean \pm standard deviation

$C A B G$ coronary artery bypass grafting, $C K D$ chronic kidney disease, $M I$ myocardial infarction, $M-P C I$ manual percutaneous coronary intervention, $P A D$ peripheral artery disease, $P C I$ percutaneous coronary intervention, $R-P C I$ robotic-assisted percutaneous coronary intervention

after manual conversion. These cases are summarized in Fig. 3.

In-hospital MACE did not occur in the two groups. There were no statistical differences in procedure time, fluoroscopy time and contrast volume between the two groups. Dose area product (DAP) and radiation exposure to the operators were significantly lower in the R-PCI (DAP: 77.6 vs. $104.8 \mathrm{Gycm}^{2}, p=0.02$, operator radiation exposure: 0 vs.

Case selection flow

$C T O$ chronic total occlusion, PCI percutaneous coronary intervention

\begin{tabular}{|c|}
\hline Robotic PCI cases \\
$2019.4 .1-2020.3 .31$ \\
30 procedures \\
28 patients \\
\hline
\end{tabular}

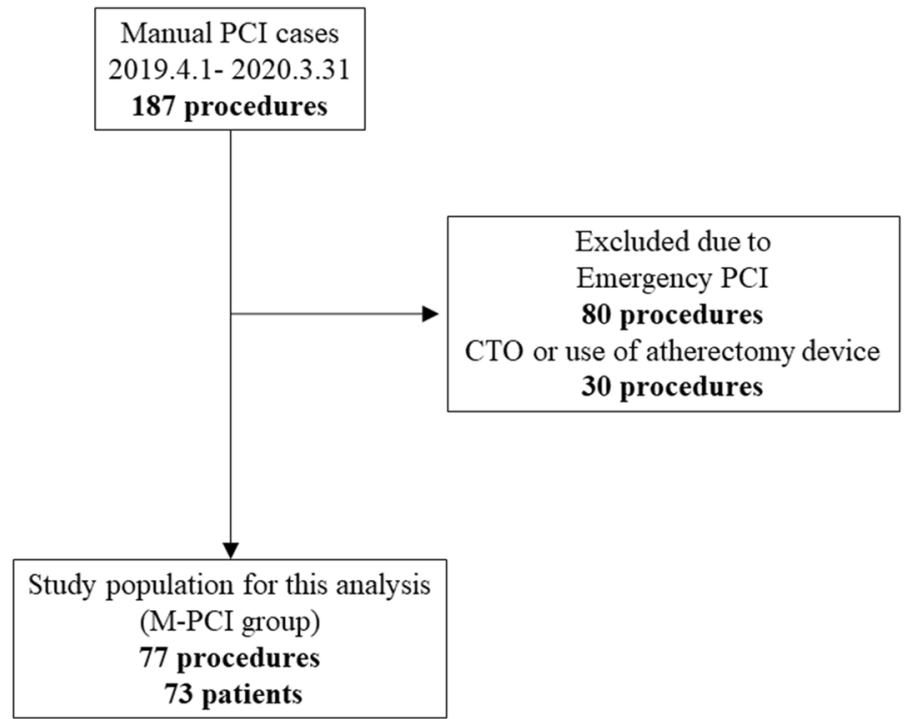


Table 2 Comparison of lesion and procedural characteristics between the R-PCI and the M-PCI

\begin{tabular}{|c|c|c|c|}
\hline & R-PCI $(L=48)$ & M-PCI $(L=109)$ & $p$ value \\
\hline \multicolumn{4}{|l|}{ Lesion background } \\
\hline Vessel & & & 0.93 \\
\hline LMT & $2.1 \%(1)$ & $6.4 \%(7)$ & \\
\hline LAD & $42.6 \%(20)$ & $44.0 \%(48)$ & \\
\hline LCX & $27.7 \%(13)$ & $22.0 \%(24)$ & \\
\hline $\mathrm{RCA}$ & $27.7 \%(13)$ & $27.5 \%(30)$ & \\
\hline Vein graft & $2.0 \%(1)$ & $0 \%$ & \\
\hline ACC/AHA classification (B2/C) & $47.9 \%(23)$ & $55.0 \%(60)$ & 0.55 \\
\hline ISR lesion & $14.6 \%(7)$ & $14.7 \%(16)$ & 1.0 \\
\hline Small vessel lesion $(\leq 2.5 \mathrm{~mm})$ & $39.6 \%(19)$ & $28.4 \%(31)$ & 0.23 \\
\hline Moderate/severe calcified lesion & $20.8 \%(10)$ & $19.3 \%(21)$ & 0.99 \\
\hline Tortuosity (<moderate) & $27.1 \%(13)$ & $20.2 \%(22)$ & 0.34 \\
\hline Diffuse lesion (> $20 \mathrm{~mm})$ & $22.9 \%(11)$ & $21.1 \%(23)$ & 0.96 \\
\hline Bifurcation lesion & $25.0 \%(12)$ & $43.1 \%(47)$ & 0.047 \\
\hline \multicolumn{4}{|l|}{ QCA analysis } \\
\hline Lesion length (mm) & $15.2 \pm 7.7$ & $14.5 \pm 9.4$ & 0.63 \\
\hline Reference diameter (mm) & $2.64 \pm 0.61$ & $2.68 \pm 0.55$ & 0.70 \\
\hline Minimum lumen diameter (mm) & $0.66 \pm 0.38$ & $0.64 \pm 0.37$ & 0.99 \\
\hline Pre $\%$ stenosis & $75.4 \pm 11.5$ & $75.4 \pm 12.3$ & 0.96 \\
\hline Post $\%$ stenosis & $14.6 \pm 13.0$ & $15.6 \pm 11.3$ & 0.64 \\
\hline \multicolumn{4}{|l|}{ Procedural background } \\
\hline Access site & & & 0.25 \\
\hline Radial & $60.4 \%(29)$ & $54.1 \%(59)$ & \\
\hline Brachial & 0 & $8.3 \%(9)$ & \\
\hline Femoral & $39.6 \%(19)$ & $37.6 \%(41)$ & \\
\hline Stenting & $73.0 \%(35)$ & $75.2 \%(82)$ & 0.91 \\
\hline Pre-dilatation & $81.3 \%(44)$ & $87.2 \%(95)$ & 0.59 \\
\hline Post-dilatation & $65.1 \%(14)$ & $40.4 \%(44)$ & 0.25 \\
\hline Intravascular imaging modality & $98.0 \%(47)$ & $99.1 \%(108)$ & 1.0 \\
\hline
\end{tabular}

All values are $\%(N)$ or mean \pm standard deviation

$A C C$ American College of Cardiology, AHA American Heart Association, ISR in-stent restenosis, LAD left anterior descending artery, $L C X$ left circumflex artery, $L M T$ left main trunk, $M-P C I$ manual percutaneous coronary intervention, $Q C A$ quantitative coronary angiography, $R C A$ right coronary artery, $R-P C I$ roboticassisted percutaneous coronary intervention
$21.5 \mu \mathrm{SV}, p<0.0001$, respectively), while radiation exposure to the assistants did not differ between the two groups (10.5 vs. $9.0 \mu \mathrm{SV}, p=0.14$ ).

\section{Propensity score-matched cohort}

After propensity score matching, 30 R-PCI procedures in 28 patients with 48 lesions and $37 \mathrm{M}$-PCI procedures in 35 patients with 45 lesions were analyzed. There were no significant differences in patients', lesion, and procedural characteristics between the two groups (Tables 4, 5). Regarding study outcomes, operator radiation exposure remained significantly lower in the R-PCI (0 vs. $24.5 \mu \mathrm{SV}, p<0.0001)$, and DAP tended to be lower in the R-PCI (77.6 vs. 100.2 $\left.\mathrm{Gycm}^{2}, p=0.07\right)$. There were no significant differences in radiation exposure to the assistants, fluoroscopy time, procedural time and contrast volume between the two groups (radiation exposure to assistants: 10.5 vs. $10.0 \mu \mathrm{SV}, p=0.64$, fluoroscopy time: 27.5 vs. $30.1 \mathrm{~min}, p=0.55$, procedural time: 72.4 vs. $61.6 \mathrm{~min}, p=0.23$, and contrast volume: 93.2 vs. $102.0 \mathrm{ml}, p=0.36$ ) (Table 6).

\section{Discussion}

The main findings of this paper are following; (1) clinical success rate with R-PCI achieved 93.3\%; (2) sixteen percent of the R-PCI procedures required partial manual assistance or manual conversion; (3) R-PCI dramatically reduced radiation exposure to operators.

This is a first report that demonstrated favorable clinical results with R-PCI in Japan. In this study, half of the target 
Table 3 Study outcomes in comparison with the R-PCI and the M-PCI

\begin{tabular}{llll}
\hline & R-PCI $(p=30)$ & M-PCI $(p=77)$ & $p$ value \\
\hline Clinical success* rate & $93.3 \%(28)$ & $92.2 \%(71)$ & 0.97 \\
Residual stenosis $<30 \%$ & $93.3 \%(28)$ & $92.2 \%(71)$ & 0.97 \\
In hospital MACE $\dagger$ & $0 \%$ & $0 \%$ & 1.0 \\
Robotic technical success $\ddagger$ rate & $90.0 \%(27)$ & & - \\
Completion robotically & $83.3 \%(25)$ & - & \\
Partial manual assistance & $6.7 \%(2)$ & & 0.40 \\
Manual conversion & $10.0 \%(3)$ & $65.6 \pm 34.8$ & 0.33 \\
Procedure time $(\min )$ & $72.4 \pm 41.2$ & $31.5 \pm 18.9$ & 0.13 \\
Fluoroscopy time $(\mathrm{min})$ & $27.5 \pm 18.9$ & $107.8 \pm 43.4$ & \\
Contrast medium $(\mathrm{ml})$ & $93.2 \pm 44.5$ & & 0.02 \\
Radiation exposure & & $104.8 \pm 54.4$ & $<0.0001$ \\
Dose area product $\left(\mathrm{Gycm}{ }^{2}\right)$ & $77.6 \pm 49.6$ & $21.5(12.0-37.5)$ & 0.14 \\
To operator $(\mu \mathrm{SV})$ & $0(0-1.3)$ & $9.0(4.0-15.0)$ & \\
To assistant $(\mu \mathrm{SV})$ & $10.5(8.8-20.3)$ & & \\
\hline
\end{tabular}

All values are $\%(N)$, mean \pm standard deviation, or median (interquartile range)

$M A C E$ major cardiac adverse event, $M-P C I$ manual percutaneous coronary intervention, $R-P C I$ roboticassisted percutaneous coronary intervention

*Clinical success: $<30 \%$ residual stenosis without in-hospital MACE

$\dagger$ MACE: a composite of a composite of cardiac death, myocardial infarction, and clinically driven target vessel revascularization

${ }^{\ddagger}$ Robotic technical success: clinical success and the completion of the PCI procedure entirely robotically or with partial manual assistance

\begin{tabular}{|c|c|}
\hline Case & Description \\
\hline \multicolumn{2}{|c|}{ Manual assistance } \\
\hline Case 1 & $\begin{array}{l}\text { A } 70 \text { 's male. Target was a severe in-stent restenosis at the mid of the left anterior descending artery. A Fielder FC guidewire (Asahi } \\
\text { Intecc.) could not pass the lesion robotically. A Gaia Next One guidewire (Asahi Intecc.) passed manually under a support of a } \\
\text { microcatheter. After re-switching to robotic procedure, the robotic procedure succeeded. }\end{array}$ \\
\hline Case 2 & $\begin{array}{l}\text { A } 60 \text { 's male. Target was a severe stenosis at the mid of the left anterior descending artery. A guidewire passed robotically and a } \\
\text { balloon catheter was delivered robotically and inflated. However, a stent could not pass the lesion. An intravascular ultrasound } \\
\text { showed that a calcified nodule existed at the proximal part of the lesion. Following additional high pressure dilatation by a non- } \\
\text { compliant balloon, the stent was delivered manually using a guide extension catheter. The procedure resulted in success. }\end{array}$ \\
\hline \multicolumn{2}{|c|}{ Manual conversion } \\
\hline Case 3 & $\begin{array}{l}\text { A } 70 \text { 's male. Target lesion was a severe in-stent restenosis at the distal of the right coronary artery. A guidewire seemed to pass the } \\
\text { lesion robotically. However, an intravascular ultrasound examination revealed the guidewire crossed a subintimal space partially. The } \\
\text { guidewire re-crossed manually using a microcatheter. The guidewire passed through a true lumen successfully. The residual part of } \\
\text { the procedure was done manually according to operator's discretion. The procedure resulted in success. }\end{array}$ \\
\hline Case 4 & $\begin{array}{l}\text { A } 70 \text { 's male. Target lesion was a sever stenosis with severe calcification at the distal of the left circumflex artery. A guidewire passed } \\
\text { robotically, but a balloon catheter could not pass. Even after switching to manual procedure, The balloon catheter could not cross } \\
\text { manually even using a guide extension catheter supporting. The procedure resulted in failure. }\end{array}$ \\
\hline Case 5 & $\begin{array}{l}\text { A } 60 \text { 's male with past history of coronary artery bypass grafting. Target was a stenosis at the distal portion of the saphenous vein graft. } \\
\text { The proximal to the stenosis was quite tortuous. A guidewire could not pass robotically. The guidewire crossed the lesion manually } \\
\text { with a support of a microcatheter. A balloon crossed manually and inflated. However, a stent could not pass the lesion even using a } \\
\text { guide extension catheter. The procedure resulted in failure. }\end{array}$ \\
\hline
\end{tabular}

Fig. 3 Description of the cases required manual assistance or manual conversion

lesions were type B2/C lesions in R-PCI. Although two $\mathrm{R}$-PCI procedures resulted in failure, overall clinical success rate with R-PCI achieved $93.3 \%$ without any in-hospital MACE. In the present study, $16.7 \%$ of the R-PCI procedures required partial manual assistance $(6.7 \%)$ due to difficulty of guidewire crossing and stent delivery, and manual conversion (10.0\%) due to difficulty of guidewire crossing and balloon catheter delivery. In the CORA-PCI study, the rates of partial manual assistance and manual conversion were $11.1 \%$ and $7.7 \%$, respectively [8]. Similarly, Harrison et al. reported that $18.5 \%$ of their R-PCI procedures required either planned partial manual assistance (3.7\%), unplanned 
Table 4 Patient characteristics in the propensity-matched cohort

\begin{tabular}{llll}
\hline & R-PCI $(n=28)$ & M-PCI $(n=35)$ & $p$ value \\
\hline Age, years & $70.9 \pm 9.9$ & $73.9 \pm 11.3$ & 0.28 \\
Male sex & $75.0 \%(21)$ & $62.9 \%(22)$ & 0.30 \\
Prior PCI & $57.1 \%(16)$ & $37.1 \%(13)$ & 0.11 \\
Prior MI & $28.6 \%(8)$ & $20.0 \%(7)$ & 0.43 \\
Prior CABG & $10.7 \%(3)$ & $2.9 \%(1)$ & 0.20 \\
Heart failure & $35.7 \%(10)$ & $28.6 \%(10)$ & 0.55 \\
Diabetes mellitus & $60.7 \%(17)$ & $51.4 \%(18)$ & 0.46 \\
Hyperlipidemia & $67.9 \%(19)$ & $77.1 \%(27)$ & 0.41 \\
Hypertension & $75.0 \%(21)$ & $88.6 \%(31)$ & 0.16 \\
Prior stroke & $14.3 \%(4)$ & $20.0 \%(7)$ & 0.55 \\
PAD & $10.7 \%(3)$ & $14.3 \%(5)$ & 0.67 \\
Current smoker & $53.6 \%(15)$ & $45.7 \%(16)$ & 0.54 \\
CKD & $28.6 \%(8)$ & $40.0 \%(14)$ & 0.34 \\
Hemodialysis & $14.3 \%(4)$ & $8.6 \%(3)$ & 0.47 \\
\hline
\end{tabular}

All values are $\%(N)$ or mean \pm standard deviation

$C A B G$ coronary artery bypass grafting, $C K D$ chronic kidney disease, $D M$ diabetes mellitus, $M I$ myocardial infarction, $M-P C I$ manual percutaneous coronary intervention, $P A D$ peripheral artery disease, $P C I$ percutaneous coronary intervention, $R-P C I$ robotic-assisted percutaneous coronary intervention

partial manual assistance (7.4\%), or manual conversion (7.4\%) due to limited guide catheter/ wire support issue or robotic platform limitations [9], which were comparable to our results. In conventional PCI, most of this kind lesions are treated under support of over-the-wire-type microcatheter or guide extension catheter which are incompatible with the current CorPath GRX system. Thus, the development of the new system which allows to manipulate microcatheters and guide extension catheters is strongly warranted for reduction of manual assistant and conversion.

Several papers have proven the reduction of radiation exposure to operator with R-PCI [6-10]. Likewise, the present study demonstrated a dramatical decrease in operator radiation exposure with R-PCI compared to M-PCI. This is an unimpeachable benefit of R-PCI. However, there have been no information on radiation exposure to assistant in R-PCI procedures. We had been concerned about an increasing assistant radiation exposure due to the absence of operator, like a "shield". In this study, the radiation exposure to assistant with R-PCI was comparable to that with M-PCI. Regarding this point, we should make further effort to reduce assistant radiation exposure more by keeping a certain distance from the X-ray generator during procedure.

A recent large-scale retrospective single-center study demonstrated the significant reduction of patients' radiation exposure with R-PCI compared to M-PCI [10]. R-PCI can provide a precise device positioning and decreased operator strain and fatigue during the procedure, which may minimize fluoroscopy times. In contrast, this study showed that there was a trend to reduce radiation exposure to patients by R-PCI, but not statistically significant. This would be attributed to our team's lack of experience and small sample size. Since R-PCI system is a novel and new technology, experience of both operator and assistant is very important. A previous study reported that R-PCI operators could reduce procedure and fluoroscopy time after three cases [11]. Thus, more experience of the operators and assistants may be able to further improve radiation exposure to both patients and assistants. To confirm the true benefit to patients, large-scale randomized R-PCI vs. M-PCI trials should be warranted. In this study, the procedural time was about 10 min longer in R-PCI than that in manual PCI, which was consistent with the previous studies $[8,10]$. This could be caused by more time in robotic drive setup and loading the robotic drive with PCI devices. The CORA-PCI demonstrated that the low complexity procedure had significantly longer procedure time with R-PCI; whereas, this would be diluted by a lengthier overall procedure time in the intermediate- and high-complexity procedures.

Recently, another potential benefit of R-PCI has been raising. In R-PCI with the present CorPath system, someone needs to be close to the table for preparation of the patient and exchange the PCI devices in the cassette. However, except those timings, all staff can stay away from the tableside; consequently, R-PCI could minimize the proximity to the patient for the majority of the procedure. Therefore, in a pandemic such COVID-19 [12,13], R-PCI may be useful to reduce the exposure risk to healthcare providers in the management of patients at high risk for COVID-19 or confirmed positives that require coronary intervention $[14,15]$.

\section{Limitations}

First, this was a retrospective study. Even though propensity sore matching was adopted to minimize differences between the two groups, a potential bias and residual confounding could not be eliminated. Second, this was a small-size and single-center study. Third, target lesions included in this study were relatively simple. To evaluate the safety and efficacy of the CorPath GRX system in the Japanese realworld practice, the nationwide post-marketing surveillance is ongoing. The results are awaited.

\section{Conclusions}

R-PCI in selected patients demonstrated favorable clinical outcomes with dramatical reduction of radiation exposure to operators in Japanese clinical practice. 
Table 5 Lesion and procedural characteristics in the propensitymatched cohort

\begin{tabular}{|c|c|c|c|}
\hline & R-PCI $(L=48)$ & M-PCI $(L=45)$ & $p$ value \\
\hline Vessel & & & 0.79 \\
\hline LMT & $2.1 \%(1)$ & $4.4 \%(2)$ & \\
\hline LAD & $41.8 \%(20)$ & $42.2 \%(19)$ & \\
\hline LCX & $27.1 \%(13)$ & $22.2 \%(10)$ & \\
\hline $\mathrm{RCA}$ & $27.1 \%(13)$ & $31.1 \%(14)$ & \\
\hline Vein graft & $2.1 \%(1)$ & & \\
\hline ACC/AHA classification (B2/C) & $50.0 \%(23)$ & $53.3 \%(24)$ & 0.60 \\
\hline ISR lesion & $14.6 \%(7)$ & $17.8 \%(8)$ & 0.78 \\
\hline Small vessel lesion $(\leq 2.5 \mathrm{~mm})$ & $39.6 \%(19)$ & $31.1 \%(14)$ & 0.52 \\
\hline Moderate/severe calcified lesion & $20.8 \%(10)$ & $17.8 \%(8)$ & 0.80 \\
\hline Tortuosity (<moderate) & $27.1 \%(13)$ & $42.4 \%(19)$ & 0.19 \\
\hline Diffuse lesion (> $20 \mathrm{~mm})$ & $22.9 \%(11)$ & $24.4 \%(11)$ & 1.0 \\
\hline Bifurcation lesion & $25.0 \%(12)$ & $33.3 \%(15)$ & 0.49 \\
\hline \multicolumn{4}{|l|}{ QCA analysis } \\
\hline Lesion length (mm) & $15.2 \pm 7.8$ & $15.5 \pm 9.6$ & 0.86 \\
\hline Reference diameter (mm) & $2.64 \pm 0.62$ & $2.64 \pm 0.60$ & 1.0 \\
\hline Minimum lumen diameter (mm) & $0.66 \pm 0.38$ & $0.66 \pm 0.44$ & 0.95 \\
\hline Pre $\%$ stenosis & $75.4 \pm 11.7$ & $73.3 \pm 18.7$ & 0.51 \\
\hline Post $\%$ stenosis & $14.6 \pm 13.2$ & $14.3 \pm 9.1$ & 0.90 \\
\hline \multicolumn{4}{|l|}{ Procedural background } \\
\hline Access site & & & 0.59 \\
\hline Radial & $60.4 \%(29)$ & $71.1 \%(32)$ & \\
\hline Brachial & 0 & $2.2 \%(1)$ & \\
\hline Femoral & $39.6 \%(19)$ & $26.7 \%(12)$ & \\
\hline Stenting & $72.9 \%(35)$ & $84.4 \%(38)$ & 0.18 \\
\hline Pre-dilatation & $91.7 \%(44)$ & $84.4 \%(38)$ & 0.28 \\
\hline Post-dilatation & $29.2 \%(14)$ & $46.7 \%(21)$ & 0.08 \\
\hline Intravascular imaging modality & $97.9 \%(47)$ & $100 \%(45)$ & 0.33 \\
\hline
\end{tabular}

All values are $\%(N)$ or mean \pm standard deviation

ACC American College of Cardiology, AHA American Heart Association, ISR in-stent restenosis, LAD left anterior descending artery, $L C X$ left circumflex artery, $L M T$ left main trunk, $M-P C I$ manual percutaneous coronary intervention, $Q C A$ quantitative coronary angiography, $R C A$ right coronary artery, $R-P C I$ roboticassisted percutaneous coronary intervention 
Table 6 Study outcomes in the propensity-matched cohort

\begin{tabular}{lllc}
\hline & R-PCI $(p=30)$ & M-PCI $(p=37)$ & $p$ value \\
\hline Clinical success * rate & $93.3 \%(28)$ & $94.6 \%(35)$ & 0.97 \\
Residual stenosis $<30 \%$ & $93.3 \%(28)$ & $94.6 \%(35)$ & 0.97 \\
In hospital MACE $\dagger$ & $0 \%$ & $0 \%$ & 1.0 \\
Robotic technical success $\ddagger$ rate & $90.0 \%(27)$ & & - \\
Completion robotically & $83.3 \%(25)$ & & \\
Partial manual assistance & $6.7 \%(2)$ & & 0.23 \\
Manual conversion & $10.0 \%(3)$ & $61.6 \pm 28.9$ & 0.55 \\
Procedure time $(\min )$ & $72.4 \pm 41.2$ & $30.1 \pm 14.9$ & 0.36 \\
Fluoroscopy time $(\mathrm{min})$ & $27.5 \pm 18.9$ & $102.0 \pm 32.9$ & \\
Contrast medium $(\mathrm{ml})$ & $93.2 \pm 44.5$ & & 0.07 \\
Radiation exposure & & $100.2 \pm 47.7$ & $<0.0001$ \\
Dose area product $\left(\mathrm{Gycm}{ }^{2}\right)$ & $77.6 \pm 49.6$ & $24.5(13.0-39.3)$ & 0.64 \\
To operator $(\mu \mathrm{SV})$ & $0(0-1.3)$ & $10.0(4.8-20.0)$ & \\
To assistant $(\mu \mathrm{SV})$ & $10.5(8.8-20.3)$ & & \\
\hline
\end{tabular}

All values are $\%(N)$, mean \pm standard deviation, or median (interquartile range)

$M A C E$ major cardiac adverse event, $M$-PCI manual percutaneous coronary intervention, $R$ - $P C I$ roboticassisted percutaneous coronary intervention

*Clinical success: $<30 \%$ residual stenosis without in-hospital MACE

${ }^{\dagger}$ MACE: a composite of a composite of cardiac death, myocardial infarction, and clinically driven target vessel revascularization

Robotic technical success: clinical success and the completion of the PCI procedure entirely robotically or with partial manual assistance

\begin{abstract}
Acknowledgements The authors gratefully acknowledge the technical assistance of Ms. Eiko ogura and the staff in the cardiac catheterization laboratory of Kurume University Hospital.
\end{abstract}

Funding This study was carried out with no external sources of funding.

\section{Compliance with ethical standards}

Conflict of interest All authors have no conflict of interest to disclose.

\section{References}

1. Roguin A, Goldstein J, Bar O (2012) Brain tumours among interventional cardiologists: a cause for alarm? Report of four new cases from two cities and a review of literature. Eurointervention 7:1081-1086

2. Vano E, Kleiman NJ, Duran A, Rehani MM, Echeverri D, Cabrera M (2010) Radiation cataract risk in interventional cardiology personnel. Radiat Res 174:490-495

3. Goldstein JA, Balter S, Cowley M, Hodgson J, Klein LW, Interventional Committee of the Society of Cardiovascular Interventions (2004) Occupational hazards of interventional cardiologists: prevalence of orthopedic health problems in contemporary practice. Catheter Cardiovasc Interv 63:407-411

4. Kagiyama K, Ueno T, Mitsutake Y, Yamaji K, Ishimatsu T, Sasaki K, Fukumoto Y (2019) First experience of robotic-assisted percutaneous coronary intervention in Japan. Intern Med 58:3415-3419

5. Moussa ID, Klein LW, Shah B, Mehran R, Mack MJ, Brilakis ES, Reilly JP, Zoghbi G, Holper E, Stone GW, Society for Cardiovascular Angiography and Interventions (2014) Consideration of a new definition of clinically relevant myocardial infarction after coronary revascularization: an expert consensus document from the Society for Cardiovascular Angiography and Interventions (SCAI). Catheter Cardiovasc Interv 83:27-36

6. Weisz G, Metzger DC, Caputo RP, Delgado JA, Marshall JJ, Vetrovec GW, Reisman M, Waksman R, Granada JF, Novack V, Moses JW, Carrozza JP (2013) Safety and feasibility of robotic percutaneous coronary intervention: PRECISE (percutaneous robotically-enhanced coronary intervention) study. J Am Coll Cardiol 61:1596-1600

7. Smitson CC, Ang L, Pourdjabbar A, Reeves R, Patel M, Mahmud E (2018) Safety and feasibility of a novel, second-generation robotic-assisted system for percutaneous coronary intervention: first-in-human report. J Invasive Cardiol 30:152-156

8. Mahmud E, Naghi J, Ang L, Harrison J, Behnamfar O, Pourdjabbar A, Reeves R, Patel M (2017) Demonstration of the safety and feasibility of robotically assisted percutaneous coronary intervention in complex coronary lesions: results of the CORA-PCI study (Complex Robotically Assisted Percutaneous Coronary Intervention). JACC Cardiovasc Interv 10:1320-1327

9. Harrison J, Ang L, Naghi J, Behnamfar O, Pourdjabbar A, Patel MP, Reeves RR, Mahmud E (2018) Robotically-assisted percutaneous coronary intervention: reasons for partial manual assistance or manual conversion. Cardiovasc Revasc Med 19:526-531

10. Patel TM, Shah SC, Soni YY, Radadiya RC, Patel GA, Tiwari PO, Pancholy SB (2020) Comparison of robotic percutaneous coronary intervention with traditional percutaneous coronary intervention: a propensity score-matched analysis of a large cohort. Circ Cardiovasc Interv 13:e008888. https://doi.org/10.1161/CIRCI NTERVENTIONS.119.008888

11. Weisz G, Smilowitz NR, Metzger DC, Caputo R, Delgado J, Marshall JJ, Vetrovec G, Reisman M, Waksman R, Pichard A, Granada JF, Moses JW, Carrozza JP (2014) The association between experience and proficiency with robotic-enhanced coronary 
intervention-insights from the PRECISE multi-center study. Acute Card Care 16:37-40

12. World Health Organization. Naming the coronavirus disease (COVID-19) and the virus that causes it. Available at https://www. who.int/emergencies/diseases/novel-coronavirus-2019/technicalguidance/naming-the-coronavirus-disease-(covid-2019)-and-thevirus-that-causes-it Accessed 20 Sept 2020

13. Moschini L, Loffi M, Regazzoni V, Di Tano G, Gherbesi E, Danzi GB (2020) Effects on QT interval of hydroxychloroquine associated with ritonavir/darunavir or azithromycin in patients with SARS-CoV-2 infection. Heart Vessels. https://doi.org/10.1007/ s00380-020-01671-4

14. Tabaza L, Virk HUH, Janzer S, George JC (2020) Robotic-assisted percutaneous coronary intervention in a COVID-19 patient. Catheter Cardiovasc Interv. https://doi.org/10.1002/ccd.28982
15. Vlachakis PK, Tentolouris A, Kanakakis I (2020) Concerns for management of STEMI patients in the COVID-19 era: a paradox phenomenon. J Thromb Thrombolysis. https://doi.org/10.1007/ s11239-020-02236-y

Publisher's Note Springer Nature remains neutral with regard to jurisdictional claims in published maps and institutional affiliations. 